\title{
Variabilidad de la frecuencia cardiaca de ciclistas de pista hombres comparado con mujeres durante un test incremental
}

\section{Heart rate variability of male versus female track cyclists during an incremental test}

\author{
Javier Porras Alvarez ${ }^{1}{ }^{*}$, Mariana Catalina Puerta Guarin ${ }^{2}$ y Fernando Javier Ávila Correa ${ }^{3}$ \\ ${ }^{1}$ Universidad Pedagógica y Tecnológica de Colombia. Tunja, Colombia \\ *Dirigir correspondencia a: javier.porras@uptc.edu.co
}

\begin{tabular}{l} 
Proceso Editorial \\
\hline Recibido: 160720 \\
Aceptado: 211220 \\
Publicado: 190221 \\
\hline
\end{tabular}

DOl 10.17081/innosa.114 (C)Copyright 2021. Porras $^{1}$ et al.

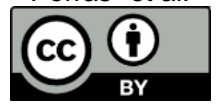

\begin{abstract}
RESUMEN
Introducción: Este estudio muestra las diferencias en la variabilidad de la frecuencia cardiaca (VFC) entre hombres y mujeres ciclistas profesionales, durante un test máximo incremental. Objetivo: Evaluar la VFC en ciclistas profesionales hombres y mujeres de pista durante un test máximo incremental de 12min. Métodos: Participaron 18 ciclistas profesionales que compiten en pruebas de pista, 7 mujeres edad: $22 \pm 5.9$ años; años de entrenamiento $5.8 \pm 2.9$ y 11 hombres edad: $21.4 \pm 4.4$ años; años de entrenamiento 6.7 \pm 3.3 . El test incremental para hombres inició con 100 vatios e incrementos de 50 vatios hasta 300 vatios, luego incrementos de 20 vatios, cada 2 minutos, cadencia pedaleo 75$90 \mathrm{rpm}$. Las mujeres iniciaron con 50 vatios, incrementos de 50 vatios hasta 200 vatios, luego incrementos de 20 vatios, cada 2 minutos, cadencia pedaleo $65-80 \mathrm{rpm}$. Resultados: Primera etapa, no existió diferencia significativa de la VFC entre los grupos. Segunda etapa, RMSSD fue mayor significativamente en las mujeres. Tercera etapa, LF/HF fue significativamente mayor en hombres. Cuarta a sexta etapa, no existió diferencia significativa en la VFC. Durante el primer minuto de recuperación, no existió diferencia significativa de la VFC. Conclusiones: Durante un test incremental en ciclistas entrenados en velocidad se observan diferencias significativas en la VFC pero a diferentes intensidades de ejercicio entre hombres y mujeres. En cambio, a la misma intensidad de ejercicio no existen diferencias significativas en la VFC, al igual que en el primer minuto de recuperación. Esto indica que en la evaluación de la VFC donde se incluyan hombres y mujeres el protocolo del test incremental debe ser exactamente el mismo.

Palabras clave: Atleta; Deporte; Sistema Cardiovascular; Sistema Nervioso autónomo.
\end{abstract}

\begin{abstract}
Introduction: This study shows the differences in heart rate variability (HRV) between men and women professional cyclists, during a maximal incremental test. Objective: To evaluate HRV in professional male and female track cyclists during a maximum incremental test of 12 min. Methods: 18 professional cyclists competing in track events participated, 7 women age: $22 \pm 5.9$ years; years of training $5.8 \pm 2.9$ and 11 men age: $21.4 \pm 4.4$ years; years of training $6.7 \pm 3$.3. The incremental test for men started with 100 watts and 50 watt increments up to 300 watts, then 20 watt increments, every 2 minutes, pedaling cadence $75-90$ rpm. Women started with 50 watts, 50 watt increments up to 200 watts, then 20 watt increments, every 2 minutes, pedaling cadence $65-80 \mathrm{rpm}$. Results: First stage, there was no significant difference in HRV between the groups. Second stage, RMSSD was significantly higher in women. Third stage, LF/HF was significantly higher in men. Fourth to sixth stage, there was no significant difference in HRV. During the first minute of recovery, there was no significant difference in HRV. Conclusions: During an incremental test in speed-trained cyclists, significant differences were observed in HRV but at different exercise intensities between men and women. On the other hand, at the same intensity of exercise there are no significant differences in HRV, as in the first minute of recovery. This indicates that the evaluation of HRV where men and women are included, the incremental test protocol must be exactly the same.

Keywords: Athlete; Autonomic Nervous System; Sport; Cardiovascular System.
\end{abstract}




\section{INTRODUCCIÓN}

El ciclismo es en la actualidad un deporte emblemático en Colombia, practicado tanto por hombres $(\mathrm{H})$ como mujeres $(\mathrm{M})$ en Ruta (carretera) y en Pista (velódromo). A nivel competitivo, las pruebas de ruta o carretera pueden durar entre tres y cinco horas o más, las pruebas de pista duran entre nueve segundos y una hora. El ciclismo de acuerdo con la clasificación de Michel Bouet (1) es un deporte atlético, clasificación sustentada en la experiencia vivida por el sujeto y referencia sobre las posibilidades motoras del ser humano; de acuerdo con la clasificación de Lev Matveiev (2) es un deporte de resistencia o cíclico, clasificación teniendo en cuenta el tipo de preparación que debe recibir el deportista a lo largo de los diferentes periodos de entrenamiento; de acuerdo con Pierre Parlebas (3) es un deporte sociomotriz con oposición contra adversarios, clasificación basada en la interacción entre el sujeto, el entorno físico y los otros participantes; de acuerdo con Mirella (4) es un deporte de resistencia de larga (Ruta) duración o velocidad (Pista) y de acuerdo con Kovacs (5) y Haskell (6) es un deporte dinámico o isotónico en función de la fisiología cardiovascular, clasificación de acuerdo con la remodelación que induce el entrenamiento y la competición sobre la función cardiaca y el corazón de los deportistas.

En el ciclismo de pista la evaluación y control del entrenamiento, así como el monitoreo del rendimiento se hace mediante un test máximo incremental, los vatios $(\mathrm{V})$ alcanzados en esta prueba se usan para determinar el máximo consumo de oxígeno $\left(\mathrm{VO}_{2} \mathrm{máx} \mathrm{ml} / \mathrm{kg} / \mathrm{min}\right)$, los umbrales ventilatorios uno y dos (VT1 \& VT2) (7-10), la frecuencia cardiaca máxima estimada (FCmáx) (11), la percepción del esfuerzo realizado (ㅁ), el umbral de lactato (UL) $(\underline{11}, \underline{12})$, la potencia media y la potencia aeróbica máxima (13-15)

El test máximo incremental es una prueba clásica donde el ciclista realiza un ejercicio en un cicloergómetro con un protocolo específico hasta el agotamiento, el cual consiste en iniciar con una carga de trabajo (vatios) entre $15 \mathrm{~V}$ y $200 \mathrm{~V}$, e ir incrementando los vatios entre 5 y $50 \mathrm{~V}$, con un tiempo determinado entre $30 \mathrm{seg}$ y $4 \mathrm{~min}$, a una cadencia de pedaleo autoseleccionada o hasta $110 \mathrm{rpm}$. La duración total de este test es de 8 a $20 \mathrm{~min}$, pero hay consenso en que la duración total para ciclistas altamente entrenados es de 8 a 12min $(\underline{13}, \underline{14}, 16,17)$, hacia los $12 \mathrm{~min}$ el ciclista no puede mantener la cadencia de pedaleo solicitada o interrumpe la prueba de forma voluntaria, por incapacidad de mantener el esfuerzo en tan altas cargas de trabajo mecánico. Por tanto, esta investigación utilizó un test con inicio de $100 \mathrm{~V}$ para hombres y $50 \mathrm{~V}$ para mujeres, de manera que a los $12 \mathrm{~min}$ los participantes interrumpieran de forma voluntaria el test por agotamiento (1835).

Los vatios obtenidos en un test máximo incremental para la evaluación y control del entrenamiento, así como el monitoreo del rendimiento también se hace mediante el análisis de variabilidad de la frecuencia cardiaca (VFC). La VFC refleja la respuesta autonómica cardiaca $(\underline{36}, \underline{37})$. Durante un test máximo incremental la VFC se puede medir de forma no invasiva latido a latido cardiaco (38). Durante el ejercicio progresivo la VFC es regulada por el incremento de la modulación simpática y retirada de la actividad parasimpática, esta respuesta cardiaca se adapta y varía de acuerdo con la intensidad del ejercicio (39-42) y con la cantidad diaria de ejercicio que realizan los atletas $(\underline{43}, \underline{44})$. El análisis de VFC se presenta como una potente herramienta 
para la evaluación y control del entrenamiento (45). Por tanto, el objetivo de esta investigación fue evaluar la VFC en ciclistas profesionales hombres y mujeres de pista durante un test máximo incremental de 12 min.

\section{MÉTODOS}

2.1. Participaron 18 ciclistas profesionales que compiten en pruebas de pista, 7 mujeres y 11 hombres, pertenecientes a la ligas de ciclismo de Boyacá y Bogotá. Teniendo en cuenta como criterio de inclusión que llevaran como mínimo tres años de entrenamiento y que hubieran participado en eventos nacionales. Los ciclistas se encontraban en el periodo preparatorio. Las pruebas se llevaron a cabo entre las 8:00 am y las 6:00 pm, en la misma ciudad pero en lugares diferentes, unos participantes realizaron el test en un laboratorio de fisiología y otros en el velódromo, por lo que pudieron existir variaciones en variables externas como humedad, temperatura, cada ciclista utilizó la bicicleta de su propiedad, excepto la rueda trasera que fue la misma para todas las pruebas y el ergómetro Tacx con tecnología bushido.

2.2. Protocolos. El test máximo incremental para hombres fue: Carga inicial de 100V, incremento de $50 \mathrm{~V}$ hasta $300 \mathrm{~V}$, a partir de esta intensidad el incremento fue de 20V, cada etapa con una duración de dos minutos, a una cadencia de pedaleo de 75-90 rpm. El test máximo incremental para mujeres fue: Carga inicial $50 \mathrm{~V}$, incremento de $50 \mathrm{~V}$ hasta $200 \mathrm{~V}$, a partir de esta intensidad el incremento fue de 20V, cada etapa con una duración de dos minutos, a una cadencia de pedaleo de 65-80 rpm (Figura 1).

Figura 1. Protocolo máximo incremental para hombres y mujeres.

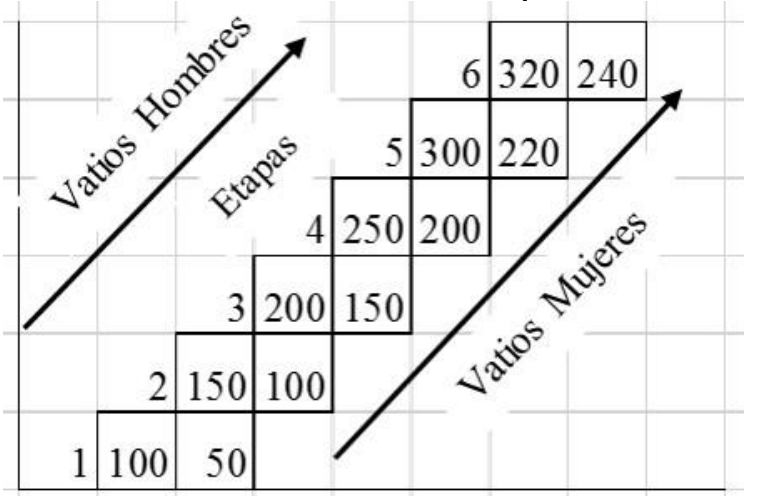

Fuente: Elaboración propia

La Figura 1 muestra los dos protocolos incrementales tanto para hombres como mujeres durante 6 etapas.

La VFC se realizó utilizando un monitor cardiaco (Polar RS800cx). El análisis de la VFC se realizó en el software Kubios HRV, versión 2.1. Los registros se tomaron latido a latido cardiaco con respiraciones espontáneas. Para el caso de las mujeres los registros se realizaron indistintamente del ciclo menstrual en el que se encontraban en el momento del test. Analizado mediante el método dominio tiempo y frecuencia, utilizando la Transformada Rápida de Fourier (FFT), de acuerdo con los criterios establecidos por la Task Force (47). Para eliminar los ruidos presentados por artefactos tecnológicos, según lo observado en los tacogramas de los intervalos RR consecutivos del latido a latido cardiaco, se aplicó el nivel "Automatic correction" de corrección de artefactos mediante el software Kubios. 
2.3. Análisis estadístico. Para evaluar el grado de asociación o independencia de la VFC en los ciclistas, se recurrió al procedimiento estadístico inferencial, comparando las medias de las distribuciones de la VFC en los Ciclistas, llevando a cabo el test T de Student. No obstante, en los casos en que no se cumplió con uno de los criterios ya sea de distribución Normal, de los parámetros de la VFC o la homogeneidad de varianzas en los grupos de ciclistas, se recurrió a la prueba estadística no paramétrica U Mann-Whitney.

2.4. Aspectos éticos. Esta investigación es clasificada como "Investigación con riesgo mínimo" según lo establecido en el artículo 11 y siguientes de la resolución 8430 de 1993 del Ministerio de Salud de Colombia (46). Ya que este estudio comparativo emplea el registro de datos a través de procedimientos comunes consistentes en exámenes físicos y evaluación de la respuesta autonómica de forma no invasiva. Todos los ciclistas firmaron un documento de consentimiento y de pleno conocimiento de procedimientos antes, durante y después del test incremental.

\section{RESULTADOS}

Las mujeres con edad: $22 \pm 5.9$ años; años de entrenamiento $5.8 \pm 2.9$ y los hombres edad: 21.4 \pm 4.4 años; años de entrenamiento $6.7 \pm 3$.3. El análisis de la VFC se realizó cada etapa de 2 minutos en el cambio de carga y durante el primer minuto de recuperación. En hombres la etapa de $340 \mathrm{~V}$ solo la terminaron tres participantes y la etapa de $360 \mathrm{~V}$ dos ciclistas únicamente. En mujeres la etapa de $260 \mathrm{~V}$ solo la terminaron dos participantes y en la de $280 \mathrm{~V}$ una participante. Por tanto, las etapas de $340 \mathrm{~V}$ y $360 \mathrm{~V}$ para hombres y las etapas de $260 \mathrm{~V}$ y $280 \mathrm{~V}$ para mujeres no se tuvieron en cuenta para el análisis.

En la Tabla 1 se presentan los resultados de la VFC en dominio tiempo y frecuencia tanto para hombres como para mujeres expresados en Media+/- DE.

Tabla 1. Parámetros de la VFC para hombres durante el test máximo incremental

\begin{tabular}{|c|c|c|c|c|c|c|c|}
\hline $\begin{array}{l}\mathbf{H} \\
\mathbf{M}\end{array}$ & $\begin{array}{l}100 v \\
50 v\end{array}$ & $\begin{array}{l}150 v \\
100 v\end{array}$ & $\begin{array}{l}200 v \\
150 v \\
\end{array}$ & $\begin{array}{l}250 v \\
200 v \\
\end{array}$ & $\begin{array}{l}300 v \\
220 \\
\end{array}$ & $\begin{array}{l}320 v(n=10) \\
240 v(n=6)\end{array}$ & $\begin{array}{l}\text { Recup } \\
1 \text { min }\end{array}$ \\
\hline \multirow[t]{2}{*}{ STD RR } & $7.6 \pm 3.3$ & $4.2 \pm 1.4$ & $2.9 \pm 1.0$ & $2.4 \pm 0.6$ & $2.3 \pm 0.6$ & $2.1 \pm 0.9$ & $2.7 \pm 0.8$ \\
\hline & $8.6 \pm 5.3$ & $5.9 \pm 2.3$ & $4.1 \pm 2.3$ & $3.5 \pm 2.1$ & $3.4 \pm 1.8$ & $3.2 \pm 1.2$ & $3.8 \pm 3.9$ \\
\hline \multirow[t]{2}{*}{ RMSSD } & $4.2 \pm 2.2$ & $3.2 \pm 1.3$ & $3.0 \pm 0.9$ & $3.1 \pm 1.0$ & $3.2 \pm 1.2$ & $3.4 \pm 1.6$ & $3.6 \pm 1.2$ \\
\hline & $5.2 \pm 1.8$ & $5.6 \pm 2.7$ & $4.1 \pm 1.5$ & $4.1 \pm 1.4$ & $4.7 \pm 2.0$ & $5.1 \pm 2.1$ & $4.6 \pm 2.6$ \\
\hline \multirow[t]{2}{*}{$\operatorname{VLF}\left(\mathrm{ms}^{2}\right)$} & $4.6 \pm 3.7$ & $1.0 \pm 0.9$ & $0.5 \pm 0.5$ & $0.4 \pm 0.5$ & $0.2 \pm 0.4$ & - & $1.2 \pm 2.4$ \\
\hline & $12.3 \pm 22.8$ & $2.6 \pm 2.5$ & $0.6 \pm 1.1$ & $1.0 \pm 2.2$ & $1.0 \pm 2.4$ & $0.2 \pm 0.4$ & $3.6 \pm 9.0$ \\
\hline \multirow[t]{2}{*}{$\operatorname{LF}\left(\mathrm{ms}^{2}\right)$} & $33.3 \pm 26.4$ & $9.6 \pm 7.4$ & $3.3 \pm 2.0$ & $0.7 \pm 1.0$ & $0.9 \pm 2.1$ & $0.1 \pm 0.3$ & $1.6 \pm 3.0$ \\
\hline & $22.9 \pm 21.7$ & $17.3 \pm 16$ & $5.6 \pm 7.6$ & $6.4 \pm 16.1$ & $10.3 \pm 25.3$ & $0.8 \pm 2$ & $24.9 \pm 64.9$ \\
\hline \multirow[t]{2}{*}{ LF/HF } & $9.6 \pm 3.9$ & $11.5 \pm 8.6$ & $9.4 \pm 4.1$ & $4.7 \pm 2.9$ & $3.7 \pm 2.5$ & $2 \pm 1.2$ & $4.6 \pm 7.3$ \\
\hline & $8.7 \pm 7.4$ & $7.1 \pm 3.8$ & $3.6 \pm 2.3$ & $4.2 \pm 2.4$ & $1.5 \pm 0.7$ & $1.5 \pm 1.2$ & $3.9 \pm 3.3$ \\
\hline \multirow[t]{2}{*}{$\mathrm{HF}\left(\mathrm{ms}^{2}\right)$} & $4.2 \pm 3.5$ & $1.0 \pm 1.0$ & $0.5 \pm 0.5$ & $0.1 \pm 0.3$ & $0.2 \pm 0.6$ & - & $0.4 \pm 0.7$ \\
\hline & $3.6 \pm 4.7$ & $4.4 \pm 6.9$ & $2.9 \pm 5.8$ & $1.0 \pm 2.6$ & $5.0 \pm 12.2$ & $0.5 \pm 1.2$ & $2.3 \pm 6$ \\
\hline \multirow[t]{2}{*}{$\begin{array}{l}\text { Total } \\
\text { power }\end{array}$} & $41.7 \pm 29.8$ & $12.0 \pm 8.3$ & $4.4 \pm 2.4$ & $1.6 \pm 1.2$ & $1.4 \pm 2.6$ & $0.3 \pm 0.5$ & $3.4 \pm 5.8$ \\
\hline & $38.7 \pm 46.9$ & $24.3 \pm 24.8$ & $9.3 \pm 13.9$ & $9.0 \pm 20.7$ & $16.5 \pm 39.9$ & $1.8 \pm 3.5$ & $30.7 \pm 79.5$ \\
\hline
\end{tabular}


STD RR: Desviación estándar de todos los intervalos R-R normales; RMSSD: Raíz cuadrada del valor medio de las sumas de las diferencias al cuadrado de todos los intervalos RR; VLF: Muy bajas frecuencias; LF: Bajas frecuencias; LF/HF: Relación entre bajas y altas frecuencias; HF: Altas frecuencias. Total: Variabilidad total. Fuente: Elaboración propia procesado en el software Kubios HRV Premium

La Tabla 2 muestra la significancia estadística en cada uno de los parámetros de la VFC de acuerdo a los vatios en cada una de las etapas del test incremental

Tabla 2. VFC y p-valor durante el test máximo incremental hombres vs mujeres

\begin{tabular}{|c|c|c|c|c|c|c|c|}
\hline $\begin{array}{l}\text { Hombres } \\
\text { Mujeres }\end{array}$ & $\begin{array}{l}100 v \\
50 v \text { P. } \\
\text { valor }\end{array}$ & $\begin{array}{l}150 v \\
100 v\end{array}$ & $\begin{array}{l}200 v \\
150 v\end{array}$ & $\begin{array}{l}250 v \\
200 v\end{array}$ & $\begin{array}{l}300 v \\
220 v\end{array}$ & $\begin{array}{l}320 v \\
240 v\end{array}$ & $\begin{array}{l}\text { Recuperación } \\
\text { 1min }\end{array}$ \\
\hline STD RR & 0.628 & $0,058 \mathrm{t}$ & $0.254 \mathrm{~W}$ & $\begin{array}{l}0.388 \\
W\end{array}$ & $\begin{array}{l}0.317 \\
W\end{array}$ & $0,062 \mathrm{t}$ & $0.578 \mathrm{~W}$ \\
\hline $\begin{array}{l}\text { RSSMD } \\
\text { (ms) }\end{array}$ & $0.331 \mathrm{t}$ & $\begin{array}{l}0,041^{*} \\
\uparrow M ~ W\end{array}$ & $0,071 \mathrm{t}$ & $\begin{array}{l}0.106 \\
t\end{array}$ & $0,059 \mathrm{t}$ & - & $0.959 \mathrm{~W}$ \\
\hline $\begin{array}{l}\text { VLF } \\
\left(\mathbf{m s}^{2}\right)\end{array}$ & $\begin{array}{l}0.890 \\
W\end{array}$ & $\begin{array}{l}0.188 \\
W\end{array}$ & $0.502 \mathrm{~W}$ & $\begin{array}{l}0.956 \\
W\end{array}$ & $1.0 \mathrm{~W}$ & - & $0.871 \mathrm{~W}$ \\
\hline $\operatorname{LF}\left(\mathrm{ms}^{2}\right)$ & $0.396 \mathrm{t}$ & $\begin{array}{l}0.203 \\
W\end{array}$ & $0.889 \mathrm{~W}$ & $\begin{array}{l}0.251 \\
t\end{array}$ & $0.226 \mathrm{t}$ & $\begin{array}{l}0.706 \\
W\end{array}$ & $0.245 \mathrm{t}$ \\
\hline LF/HF & $\begin{array}{l}0.276 \\
W\end{array}$ & $0.227 \mathrm{t}$ & $\begin{array}{l}0,003^{*} \\
\uparrow \mathrm{Ht}\end{array}$ & $\begin{array}{l}0.709 \\
t\end{array}$ & $\begin{array}{l}0,071 \\
\text { W }\end{array}$ & $0.429 \mathrm{t}$ & $0.826 \mathrm{t}$ \\
\hline $\mathrm{HF}\left(\mathrm{ms}^{2}\right)$ & $0.755 t$ & $\begin{array}{l}0,073 \\
W\end{array}$ & $0.186 \mathrm{t}$ & $\begin{array}{l}0.740 \\
W\end{array}$ & $0.200 \mathrm{t}$ & - & $0.708 \mathrm{~W}$ \\
\hline $\begin{array}{l}\text { Total } \\
\text { power }\end{array}$ & $0.868 \mathrm{t}$ & $\begin{array}{l}0.111 \\
W\end{array}$ & $0.963 \mathrm{~W}$ & $\begin{array}{l}0.249 \\
\mathrm{t}\end{array}$ & $0.217 \mathrm{t}$ & $\begin{array}{l}0.373 \\
W\end{array}$ & $0.264 t$ \\
\hline
\end{tabular}

$\uparrow$ mayor; t: prueba t; W: prueba W de Mann-Whitney; *: significancia estadística.

Fuente: Elaboración propia procesado en el software SPSS ver 15.0.

Primera etapa (100-50V HM), no hay diferencia significativa hombres comparado con mujeres en ninguno de los parámetros de la VFC. Segunda etapa (150-100V HM), RMSSD fue mayor significativamente en mujeres comparado con los hombres (Figura 2). Tercera etapa (200-150V HM), el parámetro LF/HF fue significativamente mayor en hombres comparado con las mujeres (Figura 3). Cuarta a sexta etapa, no hay diferencia significativa hombres comparado con las mujeres en ninguno de los parámetros de la VFC. Primer minuto de recuperación, no hay diferencia significativa hombres comparado con mujeres en ninguno de los parámetros de la VFC.
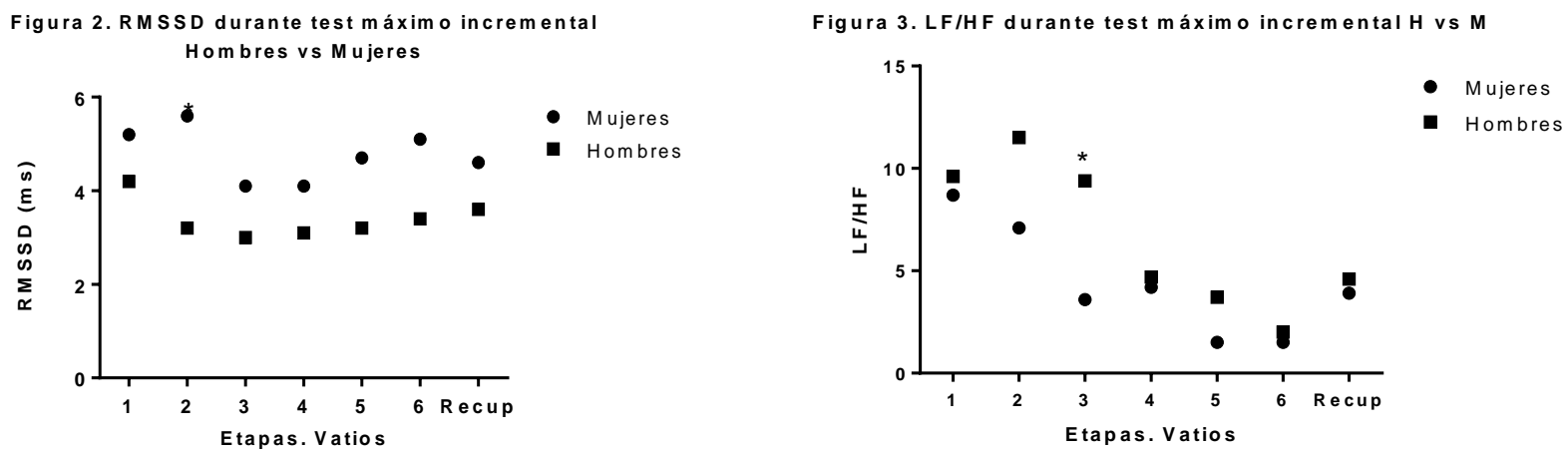
Fuente: Elaboración propia procesado en el software GraphPad Prism ver 6.0.

\section{DISCUSIÓN}

En los primeros minutos del ejercicio incremental se estima que las influencias simpáticas y parasimpáticas son aproximadamente iguales, aunque con un predominio de la actividad simpática a mayores intensidades de ejercicio. La actividad parasimpática cardiaca va disminuyendo a medida que aumenta la intensidad del ejercicio y se mantiene hasta altas intensidades de ejercicio. Sin embargo, incluso en altas intensidades de ejercicio la actividad vagal continúa modulando la función cardiaca junto con una mayor actividad simpática (4850). Los datos del presente estudio permiten evidenciar la presencia de la actividad parasimpática durante el test máximo incremental, de igual forma, se evidencia una disminución progresiva a medida que aumenta la intensidad del ejercicio.

En la primera etapa (100/50 HM), no hay diferencia significativa en hombres comparado con las mujeres en ninguno de los parámetros de la VFC, lo que indica que a baja intensidad entre los primeros $50 \mathrm{~V}$ y $100 \mathrm{~V}$ la respuesta autonómica cardiaca se comporta de la misma forma en hombres y mujeres. Al respecto se sabe que hasta los $140 \mathrm{lpm}$ las influencias simpáticas y parasimpáticas son aproximadamente iguales, cuando se comparó 100VH con 100VM, tampoco mostró diferencias significativas en ninguno de los parámetros. Consecuentemente, a bajas intensidades de ejercicio no hay diferencias en la VFC entre hombres y mujeres entrenados en ciclismo de velocidad.

No obstante, Shi \& Hu (1ㅡ) realizaron un estudio de movimiento pasivo de las extremidad inferior con una máquina que realizaba un movimiento mecánico de 5,10 y 15 ciclos por minuto en 15 hombres y 15 mujeres no entrenados en ciclismo, todos con edades entre $22.5 \pm 2.3$ años, los hombres fueron más sensibles a la respuesta del SNA (sistema nervioso autónomo) frente al movimiento pasivo de la extremidad inferior, con una actividad simpática que disminuye respecto a reposo. Por tanto, la respuesta del SNA al movimiento pasivo de las piernas está determinado por el sexo, ellos mencionan que la disminuida respuesta del SNA en las mujeres podría ser explicada por las diferencias de sexo en la sensibilidad propioceptiva, posiblemente debido a la fatiga propioceptiva articular o falla de mecanorreceptores musculares.

Sin embargo, entre los hombres se encontraron diferencias significativas dependiendo de los ciclos por minuto de movimientos pasivo de las extremidades inferiores, en cambio entre las mujeres no se encontraron diferencias significativas. Aún no está claro si la masa muscular en las extremidades inferiores puede influir en la respuesta del SNA.

Segunda etapa (150/100 HM), RMSSD fue mayor significativamente en las mujeres comparado con los hombres, esto indica que la respuesta de la actividad parasimpática a una intensidad de $150 \mathrm{~V}$ para hombres es diferente a una intensidad de $100 \mathrm{~V}$ para mujeres, porque a una mayor intensidad de ejercicio se observa un RMSSD más bajo y disminuye en función de la intensidad del ejercicio (52-54). Pero cuando se comparó RMSSD en la misma intensidad $100 \mathrm{VH}$ vs $100 \mathrm{VM}, 150 \mathrm{H}$ vs $150 \mathrm{M}$ y $200 \mathrm{VH}$ vs $200 \mathrm{VM}$, no existió diferencia significativa, 
indicando que la VFC se comporta de la misma forma tanto en hombres como mujeres en la misma intensidad de ejercicio.

Tercera etapa (200/150 HM) el parámetro LF/HF fue significativamente mayor en hombres comparado con las mujeres, cuando se comparó $200 \mathrm{VH}$ vs $200 \mathrm{VM}$ la diferencia continuó siendo significativamente mayor en hombres $p$-Valor $=0.000 . \mathrm{LF} / \mathrm{HF}$ generalmente se ha asociado con actividad simpática o equilibrio simpático-vagal" ( $\underline{55})$, sin embargo este parámetro también tiene una fuerte influencia parasimpática ( $\underline{56})$. Por tanto, este parámetro como indicador de actividad simpática o equilibrio autonómico ha sido controvertido por recientes investigaciones $(\underline{49}, \underline{50}, \underline{57})$. No obstante, como este parámetro está influenciado tanto por la actividad simpática como parasimpática, entonces a intensidades entre $150 \mathrm{~V}$ y $200 \mathrm{~V}$ existe diferencia significativa en la respuesta de la VFC entre hombres y mujeres

Cuarta a sexta etapa (250-320/200-240 HM), la actividad parasimpática en las medidas de dominio tiempo va disminuyendo cuando aumenta la intensidad del ejercicio hasta una intensidad moderada-alta, con un cambio mínimo observado a medida que la intensidad aumenta aún más $(\underline{21}, \underline{37})$, el estudio de Cottin et al., (2) mostró que durante el ejercicio incremental a intensidad por encima del umbral ventilatorio 1 (VT1) se evidencia prevalencia de actividad parasimpática en contraste con disminución de actividad simpática. Pues alrededor de la tercera y cuarta etapa está el umbral del metabolismo anaeróbico en ciclistas mujeres. Así, en el estudio de Park et al., (됴) se realizó un test discontinuo sobre siete etapas de seis minutos de duración cada una, participaron siete hombres y ocho mujeres ciclistas, todos con edades entre $30.6 \pm 5.9$ años, el umbral ventilatorio 2 (VT2) estuvo alrededor de los $200 \mathrm{~V}$ y en el estudio de Karapetian et al., (드) el umbral ventilatorio uno (VT1) estuvo alrededor de $100 \mathrm{~V}$ y $125 \mathrm{~V}$. Sin embargo, en el presente estudio no existió diferencia significativa entre hombres comparado con mujeres en los $200 \mathrm{~V}$ o $250 \mathrm{~V}$ en ninguno de los parámetros de la VFC.

El primer minuto de recuperación se considera como reactivación parasimpática (59) aunque alguna evidencia ha sugerido participación simpática también (60). En este sentido, Nakamura \& Aguiar (61) realizaron un estudio sobre el índice de reactivación cardiovascular, evaluando los primeros $30 \mathrm{seg}$ posterior al ejercicio (4min de ejercicio al $80 \%$ del umbral ventilatorio), durante fase folicular temprana y la fase media lútea del ciclo menstrual en ocho mujeres entrenadas en resistencia y 10 no entrenadas, todas con edades entre $21.6 \pm 0.2$ y $21.6 \pm 0.2$ años. Los resultados mostraron que la reactivación cardiovascular posterior al ejercicio varía según la fase del ciclo menstrual y se ve reforzada por el ejercicio aeróbico regular dependiendo de la fase del ciclo menstrual. Por tanto, la fase del ciclo menstrual se debe tener en cuenta al interpretar las funciones cardiovasculares de las mujeres. Nuestros datos se relacionan con los del estudio de McDonald (24), análisis en el cual no encontraron diferencias significativas en el primer minuto de recuperación de la frecuencia cardiaca, pero al segundo minuto sí, la prueba que realizaron fue un test máximo incremental, participaron 10 ciclistas de pista (una mujer y nueve hombres) y 15 ciclistas de ruta (4 mujeres y 11 hombres) con edades entre $25.9 \pm 6.0$ años.

La cadencia de pedaleo también puede influir en los parámetros de la VFC, Blain et al., (62) realizaron un estudio en tres diferentes cadencias de pedaleo 70, 80 y $90 \mathrm{rpm}$, participaron 15 hombres no entrenados en ciclismo, todos con edades entre $27.3 \pm 7$ años, encontraron 
diferencias en los parámetros de la VFC de 90rpm vs 70 u $80 \mathrm{rpm}$. En el presente estudio la cadencia para hombres fue de 90rpm y para mujeres fue $>65 \mathrm{rpm}$. Sin embargo, los participantes del estudio de Blain (62) no eran ciclistas entrenados, pues los ciclistas profesionales hombres se sienten muy cómodos con cadencias de pedaleo de 90-110 rpm y las mujeres con cadencias de 70-80 rpm. Finalmente, el tipo de calentamiento previo al test parece no tener ningún efecto sobre el rendimiento, en los índices de la VFC de acuerdo con los resultados encontrados por Dos-Santos et al., (르), en el que realizaron un test máximo incremental, previo a este realizaron tres tipos de calentamiento, uno sin calentamiento, dos con calentamiento y tres estiramiento balístico, en el que participaron 9 hombres no entrenados, con edades entre $22 \pm 1$ años.

\section{CONCLUSIONES}

A bajas intensidades de ejercicio entre $50 \mathrm{~V}$ y $100 \mathrm{~V}$ no hay diferencias en la VFC entre hombres y mujeres entrenados en ciclismo de velocidad durante un test máximo incremental. A intensidades entre $100 \mathrm{~V}$ para mujeres y $150 \mathrm{~V}$ para hombres la actividad parasimpática es mayor significativamente en las mujeres, pero en la misma intensidad de ejercicio (100VH vs $100 \mathrm{VM}, 150 \mathrm{VH}$ vs $150 \mathrm{VM}$ o $200 \mathrm{VH}$ vs $200 \mathrm{VM}$ ) no existe diferencia significativa durante un test máximo incremental en ciclistas entrenados en pruebas de pista.

A intensidades de $150 \mathrm{~V}$ y $200 \mathrm{~V}$ la actividad parasimpática es significativamente mayor en los hombres. A intensidades superiores a $200 \mathrm{~V}$, no hay diferencia significativa entre hombres y mujeres de pista durante un test máximo incremental, en ninguno de los parámetros de la VFC. En el primer minuto de recuperación no hay diferencia significativa en la VFC entre hombres y mujeres de pista durante un test máximo incremental. Por tanto, durante un test incremental en ciclistas de pista hay diferencias significativas en la VFC a diferentes intensidades ejercicio entre hombres y mujeres. A la misma intensidad de ejercicio no existen diferencias significativas en la VFC entre hombres y mujeres, al igual que en el primer minuto de recuperación. Esto indica que la evaluación de la VFC donde se incluyan hombres como mujeres, el protocolo del test incremental debe ser exactamente el mismo.

Contribución de los autores/Author Contributions: Los autores Javier Porras Alvarez, Fernando Javier Avila Correa y Mariana Catalina Puerta, han participado en la conceptualización; análisis formal; investigación; recursos; escritura: preparación del borrador original; escritura: revisión y edición. Todos los autores han leído y aceptado la versión publicada del manuscrito.

Fondos: Esta investigación fue financiada por la Universidad Pedagógica y Tecnológica de Colombia, Vicerrectoría de Investigaciones, Dirección de Investigaciones, CENDES. Facultad Chiquinquirá.

Agradecimientos: Los autores expresan su agradecimiento a los ciclistas y entrenadores de las ligas de ciclismo de pista de los departamentos de Boyacá y Bogotá. Al equipo de JenaroLab. Al Programa en extensión Licenciatura en Educación Física, Recreación y Deporte. Facultad Chiquinquirá. Proyecto "Respuesta autonómica durante el ejercicio de mujeres versus hombres entrenados en Ciclismo de velocidad". Código SGI: 2136. Grupo de investigación FIMED. 
Conflictos de intereses: "Los autores declaran no tener ningún conflicto de intereses, ya sea entre los autores de la investigación y el director del estudio u otros"

\section{REFERENCIAS}

1. Bouet M. Signification du sport. 1968 [cited 2020 Jun 24] http://archives.umc.edu.dz/handle/123456789/113451

2. Matveev LP. Periodización del entrenamiento deportivo [Internet]. Madrid: IN. 1975 [cited 2020 Jul

https://books.google.com.co/books?id=arTbAAAACAAJ\&dq=Periodización+del+entrenamiento +deportivo.\&hl=es\&sa=X\&ved=2ahUKEwjuwcGhpM3qAhUjhOAKHfqWABoQ6AEwAHoECAA QAQ

3. Parlebas P. Contribution a un léxique commenté en science de laaction motrice. 198.

4. Mirella R. Las nuevas metodologías del entrenamiento de la fuerza, la resistencia, la velocidad y la flexibilidad [Internet]. Primera. Editorial Paidotribo; 2006 [cited 2020 Jul 14].https://www.google.com/search?biw=1366\&bih=608\&tbm=bks\&ei=bu0NX-

7SMqOlggf6rYLQAQ\&q=Las+nuevas+metodologías+del+entrenamiento+de+la+fuerza\%2C+l a+resistencia\%2C+la+velocidad+y+la+flexibilidad\&oq=Las+nuevas+metodologías+del+entren amiento+de+la+fuerza\%2C+la+r

5. Kovacs $R$, medicine AB-T in cardiovascular, 2016 undefined. Cardiovascular adaptation in athletes. Elsevier [Internet]. [cited $2020 \quad$ Jul https://www.sciencedirect.com/science/article/pii/S1050173815001188?casa token=6iVaa7sSCsAAAAA:fg yro3j30iivEOoHG2QG9KSC xLZM6iLeIMXQL7vHhQBYKouobp CbGMhM18qlJcgSv2NaBw

6. Borg G. Perceived exertion. Exerc Sport Sci [Internet]. 1974 [cited 2020 Jun 24]; https://journals.Iww.com/acsm-essr/Citation/1974/00020/Perceived Exertion.6.aspx

7. Cucullo JM, Terreros JL, Layus F, Quilez J. Prueba ergométrica indirecta. ¡Metodología para el cálculo óptimo de VO2 máx. en ciclistas RESUMEN RESUME [Internet]. apunts.org. [cited 2020 Jun 24]. $\quad$ https://www.apunts.org/index.php?p=revista\&tipo=pdfsimple\&pii=X0213371787049770

8. Duncan G, Howley E, in BJ-M and science, 1997 undefined. Applicability of VO2max criteria: discontinuous versus continuous protocols. europepmc.org [Internet]. [cited 2020 Jul 14] https://europepmc.org/article/med/9044234

9. Lucía A, Rabadán M, Hoyos J, Hernández-Capilla M, Pérez M, San Juan AF, et al. Frequency of the $\dot{V} O 2 m a x$ plateau phenomenon in world-class cyclists. Int J Sports Med [Internet]. 2006 Dec 30 [cited 2020 Jul 14];27(12):984-92. http://www.thieme-connect.de/DOI/DOI?10.1055/s$\underline{2006-923833}$

10. Lee J-M, Bassett DR, Thompson DL, Fitzhugh EC. Validation of the Cosmed Fitmate for Prediction of Maximal Oxygen Consumption. J Strength Cond Res [Internet]. 2011 Sep [cited 2020 Jul 14];25(9):2573-9. http://journals.Iww.com/00124278-201109000-00029

11. Casajús JA, Piedrafita E, Aragonés M T. CRITERIOS DE MAXIMALIDAD EN PRUEBAS DE ESFUERZO CRITERIA FOR MAXIMAL EXERCISE TEST. redalyc.org [Internet]. 2009 [cited 2020 Jun 24];9(35):217-31. https://www.redalyc.org/pdf/542/54223022001.pdf

12. Hill A, Medicine HL-QAIJ of, 1923 undefined. Muscular exercise, lactic acid, and the supply and utilization of oxygen. academic.oup.com [Internet]. [cited 2020 Jul 14]; https://academic.oup.com/ajmed/article-abstract/os-16/62/135/1594478

13. Faria EW, Parker DL, Faria IE. The science of cycling: Physiology and training - Part 1. Vol. 35 , Sports Medicine. 2005. p. 285-312. 
14. Faria E, Parker D. The science of cycling: factors affecting performance--Part 2. go.gale.com [Internet]. $\quad$ [cited $2020 \quad$ Jul https://go.gale.com/ps/i.do?id=GALE\%7CA200844007\&sid=googleScholar\&v=2.1\&it=r\&linkacc ess=abs\&issn $=01121642 \& p=A O N E \& s w=w$

15. Atkinson G, Davison R, ... AJ-J of sports, 2003 undefined. Science and cycling: current knowledge and future directions for research. Taylor Fr [Internet]. [cited 2020 Jun 24] https://www.tandfonline.com/doi/abs/10.1080/0264041031000102097

16. Bezerra De Almeida M, Ricardo DR, Araujo CG. Variabilidade da frequência cardíaca em um teste verdadeiramente máximo [Internet]. researchgate.net. [cited 2020 Jul 14]. https://www.researchgate.net/publication/200138055

17. Bentley RF, Vecchiarelli E, Banks L, Gonçalves PEO, Thomas SG, Goodman JM. Heart rate variability and recovery following maximal exercise in endurance athletes and physically-active individuals. Appl Physiol Nutr Metab [Internet]. 2020 Apr 15 [cited 2020 Jul 14];apnm-2020-0154. http://www.nrcresearchpress.com/doi/10.1139/apnm-2020-0154

18. Cottin F, Leprêtre PM, Lopes P, Papelier Y, Médigue C, Billat V. Assessment of ventilatory thresholds from heart rate variability in well-trained subjects during cycling. Int J Sports Med. 2006 Dec;27(12):959-67.

19. Anosov O, Patzak A, Kononovich Y, Persson PB. High-frequency oscillations of the heart rate during ramp load reflect the human anaerobic threshold. Eur J Appl Physiol. 2000;83(4-5):38894.

20. Lucia A, Hoyos J, science JC-M and, 2001 undefined. Preferred pedalling cadence in professional cycling. pdfs.semanticscholar.org [Internet]. [cited 2020 Jul 14]. https://pdfs.semanticscholar.org/9202/27402213d7684f1a18d47bc80d0543bacb77.pdf

21. Karapetian GK, Engels HJ, Gretebeck RJ. Use of heart rate variability to estimate LT and VT. Int J Sports Med [Internet]. 2008 Aug 22 [cited 2020 Jul 14];29(8):652-7. http://www.thiemeconnect.de/DOI/DOI?10.1055/s-2007-989423

22. Cottin FO, Digue CM, Lepre^tre P-M, Lepre^tre L, Papelier Y, Koralsztein J-P, et al. Heart Rate Variability during Exercise Performed below and above Ventilatory Threshold. researchgate.net [Internet]. 2004 [cited 2020 Jun 24]. http://www.acsm-msse.org

23. Bishop D, Jenkins D. The relationship between plasma lactate parameters, Wpeak and 1-h cycling performance in women. europepmc.org [Internet]. 1998 [cited 2020 Jun 24]; https://europepmc.org/article/med/9710868

24. Mcdonald KG, Grote S, Shoepe TC. Accepted for printing in. J Hum Kinet [Internet]. 2014 [cited 2020 Jul 14];41:43-9. http://www.johk.pl/

25. Moseley L, In AJ. The reliability of cycling efficiency. Med Sci [Internet]. 2001 [cited 2020 Jul 14]; https://pdfs.semanticscholar.org/eed4/f50eee1600210eb325bb71d0e867dd3ab504.pdf

26. Heil D, Wilcox A, Quinn CM. Cardiorespiratory responses to seat-tube angle variation during steady-state cycling. Exerc - Sci Sport [Internet]. 1995 [cited 2020 Jul 14]; https://europepmc.org/article/med/7674878

27. Bentley D, Wilson G, Davie A. Correlations between peak power output, muscular strength and cycle time trial performance in triathletes. he J Sport [Internet]. 1998 [cited 2020 Jul 14] https://europepmc.org/article/med/9830826

28. Fernández-García B, Pérez J. Intensity of exercise during road race pro-cycling competition. Landaluce - Sci [Internet]. 2000 [cited 2020 Jul 14]; https://europepmc.org/article/med/10795793

29. Laursen PB, Jenkins DG. The scientific basis for high-intensity interval training: Optimising training programmes and maximising performance in highly trained endurance athletes. Vol. 32, Sports Medicine. Adis International Ltd; 2002. p. 53-73.

30. MARSH A, MARTIN P. Effect of cycling experience, aerobic power, and power output on preferred and most economical cycling cadences. Med Sci Sport Exerc [Internet]. 1997 [cited 2020 Jul 14]. https://insights.ovid.com/mespex/199709000/00005768-199709000-00016 
31. MacRae H, Hise K, Allen P. Effects of front and dual suspension mountain bike systems on uphill cycling performance. Med Sci Sports [Internet]. 2000 [cited 2020 Jul 14]; https://europepmc.org/article/med/10912893

32. Dos-Santos R da CMCCR di MFB da SAL. Effects of Pre-Exercise Activities on Progressive Cycling Test Performance and Autonomic Response. 2014 Oct [cited 2020 Jul 14];17(5):84-94. https://www.asep.org/asep/asep/JEPonlineOCTOBER2014 Silveira.pdf

33. Lepers R, Maffiuletti N. Evidence of neuromuscular fatigue after prolonged exercise Mode of muscle contraction and corticospinal excitability View project Blood flow stimulation and Performance recovery View project. 2000 [cited 2020 Jul 14];1880-6. https://www.researchgate.net/publication/12247282

34. Blain G, Meste O, Bouchard T, Blain G, De Toulon-Var U. Assessment of ventilatory thresholds during graded and maximal exercise test using time varying analysis of respiratory sinus arrhythmia. Br J Sport Med [Internet]. 2005 [cited 2020 Jul 14];39:448-52. www.bjsportmed.com

35. HARNISH CR, SWENSEN TC, PATE RR. Methods for estimating the maximal lactatesteady state in trained cyclists. Med Sci Sport Exerc [Internet]. 2001 Oct [cited 2020 Jul 14];1052-5. https://www.researchgate.net/profile/Chris Harnish/publication/11934457 Methods for estima ting the maximal lactate steady state in trained cyclists/links/5a0e070daca27244d285870 e/Methods-for-estimating-the-maximal-lactate-steady-state-in-trained-cyclists.pdf

36. Gourine A V., Ackland GL. Cardiac vagus and exercise [Internet]. Vol. 34, Physiology. American Physiological Society; 2019 [cited 2020 Jul 15]. p. 71-80. www.physiologyonline.org

37. Michael S, Graham KS, Oam GMD. Cardiac autonomic responses during exercise and postexercise recovery using heart rate variability and systolic time intervals-a review [Internet]. Vol. 8, Frontiers in Physiology. Frontiers Media S.A.; 2017 [cited 2020 Jul 15]. p. 301. www.frontiersin.org

38. GAMELIN FX, BERTHOIN S, BOSQUET L. Validity of the Polar S810 Heart Rate Monitor to Measure R-R Intervals at Rest. Med Sci Sport Exerc [Internet]. 2006 May [cited 2020 Jun 16];38(5):887-93. http://journals.Iww.com/00005768-200605000-00013

39. Grant CC, Mongwe L, Janse Van Rensburg DC, Fletcher L, Wood PS, Terblanche E, et al. The difference between exercise-induced autonomic and fitness changes measured after 12 and 20 weeks of medium-to-high intensity military training. J Strength Cond Res. 2016 Sep $1 ; 30(9): 2453-9$.

40. Kaikkonen P, Hynynen E, Mann T, ... HR-E journal of, 2012 undefined. Heart rate variability is related to training load variables in interval running exercises. Springer [Internet]. [cited 2020 Jun 27]. https://link.springer.com/content/pdf/10.1007/s00421-011-2031-z.pdf

41. Seiler S, Haugen O, Kuffel E. Autonomic recovery after exercise in trained athletes: Intensity and duration effects. Med Sci Sports Exerc. 2007 Aug;39(8):1366-73.

42. Casties JF, Mottet D, Le Gallais D. Non-linear analyses of heart rate variability during heavy exercise and recovery in cyclists. Int J Sports Med [Internet]. 2006 Oct 1 [cited 2020 Jul 14];27(10):780-5. http://www.thieme-connect.de/DOI/DOI?10.1055/s-2005-872968

43. Sartor F, Vailati E, Valsecchi V, ... FV-TJ of, 2013 undefined. Heart rate variability reflects training load and psychophysiological status in young elite gymnasts. journals.Iww.com [Internet]. [cited 2020 Jun 26]; https://journals.Iww.com/nscajscr/fulltext/2013/10000/Heart Rate Variability Reflects Training Load and.19.aspx

44. Plews DJ, Laursen PB, Stanley J, Kilding AE, Buchheit M. Training adaptation and heart rate variability in elite endurance athletes: Opening the door to effective monitoring. Vol. 43, Sports Medicine. 2013. p. 773-81.

45. Porras-Alvarez J, Olinda Bernal-Calderón M. Variabilidad de la frecuencia cardiaca: evaluación del entrenamiento deportivo. Revisión de tema Heart rate variability: sports training evaluation. Theme review. Duazary [Internet]. 2019 [cited 2020 Jul 15];16(2):259-69. http://orcid.org/0000$\underline{00001-8929-7530}$ 
46. Colombia RN-R de. 8430 de 1993 del Ministerio de Salud.

47. Circulation TF-, 1996 undefined. of measurement, physiological interpretation and clinical use.

48. Gourine A V., Ackland GL. Cardiac vagus and exercise. Vol. 34, Physiology. American Physiological Society; 2019. p. 71-80.

49. White DW, Raven PB. Autonomic neural control of heart rate during dynamic exercise: Revisited. Vol. 592, Journal of Physiology. Blackwell Publishing Ltd; 2014. p. 2491-500.

50. Michael S, Graham KS, Oam GMD. Cardiac autonomic responses during exercise and postexercise recovery using heart rate variability and systolic time intervals-a review. Vol. 8, Frontiers in Physiology. Frontiers Media S.A.; 2017.

51. Hu S, Shi P, Yu H. The response of the autonomic nervous system to passive lower limb movement and gender differences. Springer [Internet]. 2015 Aug 1 [cited 2020 Jul 15];54(8):1159-67. https://www.researchgate.net/publication/281371940

52. Karapetian GK, Engels HJ, Gretebeck KA, Gretebeck RJ. Effect of caffeine on LT, VT and HRVT. Int J Sports Med. 2012;33(7):507-13.

53. Kaikkonen P, Rusko H, Martinmäki K. Post-exercise heart rate variability of endurance athletes after different high-intensity exercise interventions. Scand J Med Sci Sport. 2008 Aug;18(4):5119.

54. Hautala AJ, Mäkikallio TH, Seppänen T, Huikuri H V., Tulppo MP. Short-term correlation properties of $\mathrm{R}-\mathrm{R}$ interval dynamics at different exercise intensity levels. Clin Physiol Funct Imaging. 2003;23(4):215-23.

55. Teixeira L, Ritti-Dias RM, Tinucci T, Mion D, De Moraes Forjaz CL. Post-concurrent exercise hemodynamics and cardiac autonomic modulation. Eur J Appl Physiol [Internet]. 2011 Sep 23 [cited 2020 Jun 26];111(9):2069-78. https://link.springer.com/article/10.1007/s00421-010-18111

56. CACIOPPO JT, BERNTSON GG, BINKLEY PF, QUIGLEY KS, UCHINO BN, FIELDSTONE A. Autonomic cardiac control. II. Noninvasive indices and basal response as revealed by autonomic blockades. Psychophysiology. 1994;31(6):586-98.

57. Billman GE. The effect of heart rate on the heart rate variability response to autonomic interventions. Front Physiol. 2013;4 AUG.

58. Park SW, Brenneman M, Cooke WH, Cordova A, Fogt D. Determination of Anaerobic Threshold by Heart Rate or Heart Rate Variability using Discontinuous Cycle Ergometry. Int J Exerc Sci [Internet]. 2014 [cited 2020 Jul 15];7(1):45-53. http://www.ncbi.nlm.nih.gov/pubmed/27182400

59. Peçanha T, Silva-Júnior ND, Forjaz CL de M. Heart rate recovery: autonomic determinants, methods of assessment and association with mortality and cardiovascular diseases. Clin Physiol Funct Imaging [Internet]. 2014 Sep 1 [cited 2020 Jun 27];34(5):327-39. http://doi.wiley.com/10.1111/cpf.12102

60. Pichon A, De Bisschop C, Roulaud M, Denjean A. Spectral Analysis of Heart Rate Variability during Exercise in Trained Subjects. researchgate.net [Internet]. 2004 [cited 2020 Jul 15]. https://www.researchgate.net/publication/8134523

61. Nakamura FY, Fernando Aguiar A. Alteração do limiar de variabilidade da freqüência cardíaca após treinamento aeróbio de curto prazo [Internet]. periodicos.rc.biblioteca.unesp.br. [cited 2020 Jun 16]. https://www.researchgate.net/publication/228944567

62. Blain G, Meste O, Blain A, Bermon S. Time-frequency analysis of heart rate variability reveals cardiolocomotor coupling during dynamic cycling exercise in humans. Am J Physiol - Hear Circ Physiol. 2009 May;296(5). DOI: 10.1152/ajpheart.00881.2008 